\title{
Multiple Roles of Sms2 in White and Brown Adipose Tissues from Diet- induced Obese Mice
}

\author{
Hisatoshi Hanamatsu ${ }^{1}$, Susumu Mitsutake ${ }^{2}$, Shota Sakai ${ }^{1}$, Toshiro Okazaki ${ }^{3}$, Ken Watanabe ${ }^{4}$, Yasuyuki Igarashi ${ }^{1}$ and Kohei Yuyama $^{1 *}$ \\ ${ }^{1}$ Lipid Bio function Section, Faculty of Advanced Life Science, Hokkaido University, Sapporo, Japan \\ ${ }^{2}$ Department of Applied Biochemistry and Food Science, Faculty of Agriculture, Saga University, Saga, Japan \\ ${ }^{3}$ Department of Hematology/Immunology, Kanazawa Medical University, Ishikawa, Japan \\ ${ }^{4}$ Department of Bone and Joint Disease, National Center for Geriatrics and Gerontology, Aichi, Japan
}

*Corresponding author: Kohei Yuyama, Lipid Biofunction Section, Faculty of Advanced Life Science, Hokkaido University, Sapporo, Japan, Tel: +81-(11)-706-9047; Email: kyuyama@pharm.hokudai.ac.jp

Received Date: July 17, 2018; Accepted Date: July 28, 2018; Published Date: August 03, 2018

Copyright: ( 2018 Hanamatsu H, et al. This is an open-access article distributed under the terms of the Creative Commons Attribution License, which permits unrestricted use, distribution, and reproduction in any medium, provided the original author and source are credited.

\begin{abstract}
Background/Objectives: Adipose tissue (AT) has an important role in energy homeostasis. The dysfunction of AT or the hyper-accumulation of neutral lipids leads to various metabolic diseases. Recent studies indicate that sphingolipid metabolism associates with the development of metabolic diseases. Sphingomyelin, a major sphingolipid in mammals, requires sphingomyelin synthase (SMS) for biosynthesis. Previously, we reported that Sms2 deficiency inhibited diet-induced obesity, fatty liver, and insulin resistance in mice. However, the contribution of SMS2 to obesity and insulin resistance in AT is largely unknown. In this study, we investigated whether Sms2 deficiency in ATs affects obesity and insulin resistance.
\end{abstract}

Subjects/Methods: Wild-type and Sms2 knockout (KO) mice were fed a high-fat for 12 weeks. Body and AT weights, and the food intake, were recorded. The AT status and macrophage infiltration were evaluated by histological analysis. The expression levels of genes and proteins involved in adipogenesis, inflammation, energy expenditure, and fatty acid metabolism were examined.

Results: In white adipose tissue (WAT) from Sms2 KO mice, the number of small adipocytes increased but the adipocyte size decreased. In epididymal WAT, Sms2 deficiency inhibited inflammation and macrophage infiltration. Moreover, adipogenesis was moderately suppressed. In subcutaneous WAT from Sms2 KO mice, the expression of genes involved in energy expenditure and browning (Ucp1, Cidea, Tbx1) was elevated. In brown adipose tissue (BAT) from Sms2 KO mice, the lipid droplet surface area was lower than that of WT mice and the expression of genes involved in fatty acid synthesis (Fasn, Scd1) decreased.

Conclusion: These results demonstrate that $S m s 2$ deficiency leads to moderate adipogenesis and inflammatory suppression in epididymal WAT, increased energy expenditure by the browning of subcutaneous WAT, and suppression of fatty acid synthesis in BAT, suggesting that these synergetic effects in ATs from Sms 2 KO mice contribute to the suppression of diet-induced obesity and insulin resistance.

Keywords: Sphingomyelin synthase; Diet-induced obesity; White adipose tissue; Brown adipose tissue; Beige adipocyte

\section{Introduction}

Obesity, a central factor for metabolic syndrome, is caused by the accumulation of neutral lipids in adipose tissues. Based on its structural and functional characteristics, adipose tissue can be classified as white adipose tissue (WAT) or brown adipose tissue (BAT). WAT is a storage organ that converts excess supply calories into neutral lipids. In addition, the neutral lipid storing function of WAT is critical for preventing the accumulation of neutral lipids in other nonadipose tissues, which can result in tissue lipotoxicity [1,2]. However, the hypertrophy of white adipocytes caused by the accumulation of neutral lipids results in the onset of low-grade inflammation in WAT and the induction of systemic insulin resistance. In contrast to WAT, BAT is an energy-consumption organ that metabolizes glucose and fatty acids, and generates heat via mitochondrial uncoupling protein 1
(UCP1) $[3,4]$. Recently, brown-like adipocytes, defined as beige or brite cells that express UCP1, were identified in WAT. These cells were found to possess thermogenic properties that resemble brown adipocytes $[5,6]$ Thus, the suppression of inflammation in WAT and the enhancement of energy expenditure in WAT and BAT are expected to be a promising strategy for decreasing obesity and its related diseases.

Sphingolipids, such as ceramide (Cer) and sphingosine, are bioactive lipids that function in membrane structure stabilization, cellto-cell recognition, and cell signaling $[7,8]$. Sphingolipid metabolism involves several biomolecules, and is regulated by many enzymes [9]. It was recently reported that a dysfunction in sphingolipid metabolism is responsible for the pathogenesis of several diseases such as cancer, Alzheimer' disease, and metabolic syndrome [10,11] Sphingomyelin (SM), one of the most abundant sphingolipids in mammalian cell membranes, is iosynthesized from Cer and phosphatidylcholine with sphingomyelin synthase (SMS). SMS has three isoforms, SMS1, SMS2, and SMSr. SMS1 localizes to the Golgi apparatus, where it produces an 
Page 2 of 8

abundant supply of SM. SMSr localizes to the endoplasmic reticulum and synthesizes ceramide phosphoethanolamine, but not SM. SMS2 predominantly localizes to the plasma membrane, and it maintains SM levels in lipid rafts [12-14]. A recent study reported that Sms1 knockout (KO) mice exhibit systemic loss of adipose tissue mass, and that SMS1 is important for the maintenance of adipose tissue function by controlling oxidative stress $[15,16]$. We previously reported that Sms2 KO mice exhibited resistance against diet-induced obesity, glucose intolerance, and fatty liver [17]. However, it is unclear how diet-induced obesity and insulin resistance are suppressed in Sms2 KO mice. In addition, the role of SMS2 in adipose tissues has not been established. In this study, we investigated the effects of $S m s 2$ deficiency on the functions of WAT and BAT from Sms2 KO mice. Our results indicate that Sms2 deficiency in adipose tissues contributes to the suppression of diet-induced obesity and insulin resistance.

\section{Materials and Methods}

\section{Animals and diets}

Constitutive Sms 2 KO mice were generated as previously described [17]. To induce obesity, age- and sex-matched WT and Sms2 KO mice at 4 weeks of age were fed a high-fat diet $(60 \% \mathrm{kcal}$ from fat; $58 \mathrm{Y} 1$, TestDiet, Richmond, IN, USA) for 12 weeks. Control mice received a standard chow diet (AIN76A, TestDiet). All mice had access to water and food ad libitum, and were housed under $12 \mathrm{~h}$ light and dark cycles in a temperature-controlled room.

The body weights of mice were recorded once a week. The daily food intake was recorded in mice at 8 weeks of age. Rectal temperature was measured at dark cycles using animal thermometer (Natsume Seisakusho, Tokyo, Japan). All experiments were approved by the Animal Care Committee of Hokkaido University.

\section{Histological analysis}

Fresh WAT and BAT were fixed in $10 \%$ formaldehyde and embedded in optimal cutting temperature compound for cryosectioning. The adipose tissue cross-sections were stained with hematoxylin and eosin. The Sapporo General Pathology Laboratory (Hokkaido, Japan) performed these procedures. The cross-sections were examined under an inverted Olympus CKX41 microscope (Center Valley, PA, USA), and the images were analyzed with ImageJ software (National Institutes of Health, Bethesda, MD, USA). The frequency distribution of the adipocyte surface area was analyzed with SAS (version 2.0; Esumi, Tokyo, Japan).

\section{RNA extraction and quantitative real-time polymerase chain reaction}

Total RNA was extracted from adipose tissue and differentiated cells using TRIzol reagent (Invitrogen, Carlsbad, CA, USA) and purified with the PureLink RNA Mini Kit (Invitrogen). To synthesize singlestranded cDNA, reverse transcription was performed using the PrimeScript RT Master Mix (TAKARA Bio, Otsu, Japan) according to the instructions provided by the manufacturer. Quantitative real-time polymerase chain reaction (PCR) was performed using SYBR Premix EX Taq (TAKARA Bio) and the Thermal Cycler Dice Real-Time System (TAKARA Bio). The primers used for quantitative real-time PCR are listed in Supplementry Table 1. The gene expression levels were normalized to hypoxanthine phosphoribosyltransferase (Hprt1) or TATA box-binding protein (Tbp1) in adipose tissue or differentiated cells, respectively.

\begin{tabular}{|l|l|l|l|l|}
\hline \multirow{2}{*}{} & \multicolumn{2}{l|}{} & \multicolumn{2}{l|}{ HFD } \\
\cline { 2 - 5 } & ND & KO & WT & KO \\
\hline Epi WAT (g) & $0.78 \pm 0.16$ & $0.36 \pm 0.16^{*}$ & $2.70 \pm 0.22$ & $1.11 \pm 0.53^{* *}$ \\
\hline Ing WAT (g) & $0.61 \pm 0.16$ & $0.27 \pm 0.14^{*}$ & $2.09 \pm 0.28$ & $0.74 \pm 0.37^{* *}$ \\
\hline BAT (g) & $0.09 \pm 0.02$ & $0.06 \pm 0.01^{*}$ & $0.15 \pm 0.03$ & $0.08 \pm 0.02^{* *}$ \\
\hline Liver (g) & $0.86 \pm 0.07$ & $0.84 \pm 0.16$ & $0.96 \pm 0.19$ & $0.74 \pm 0.1$ \\
\hline Kidney (g) & $0.33 \pm 0.02$ & $0.32 \pm 0.02$ & $0.37 \pm 0.02$ & $0.34 \pm 0.03$ \\
\hline $\begin{array}{l}\text { Values are given as mean } \pm \text { S.D. The significance of differences between the WT and KO within the same condition was analyzed by Student's t-test. ( } \mathrm{n}=11-13 ; \\
\left.\text { Adipose tissue, } \mathrm{n}=3-8 ; \text { Liver and kidneys, }{ }^{*}, 0.01<p<0.05,{ }^{* *}, \mathrm{p}<0.01 .\right)\end{array}$ \\
\hline
\end{tabular}

Table 1: Tissue weight of WT and Sms2 KO mice fed ND and HFD at 15 weeks of age.

\section{Isolation and differentiation of stromal vascular cells}

Stromal vascular cells (SVCs) were isolated and cultured to induce differentiation using the method of Sato et al. with minor modifications [18]. Epididymal adipose tissues from WT and Sms2 KO mice at 6 weeks of age were resected and washed with Dulbecco's Modified Eagle Medium/Nutrient Mixture F12 (DMEM/F12, Sigma, St. Louis, MO, USA), followed by rinsing with Hanks' Balanced Salt Solution (HBSS, Invitrogen). The isolated adipose tissue was minced with scissors and digested with $0.2 \%$ collagenase (Sigma) and $0.5 \%$ bovine serum albumin (Fraction V, Sigma) in DMEM/F12. After digestion for $1 \mathrm{~h}$ at $37^{\circ} \mathrm{C}$, the suspension was filtered through a $100 \mu \mathrm{m}$ cell strainer (Corning, Corning, NY, USA) and centrifuged at $800 \times \mathrm{g}$ for $5 \mathrm{~min}$. The resulting pellet was suspended in DMEM/F12 containing $1 \%$ fetal bovine serum (FBS) and centrifuged at $800 \times \mathrm{g}$ for $5 \mathrm{~min}$. Finally, the resulting pellet was suspended in DMEM/F12 containing $10 \%$ FBS and centrifuged at $800 \times \mathrm{g}$ for $5 \mathrm{~min}$. The cells were counted. To induce adipocyte differentiation, the cells were maintained in Visceral Adipocyte Culture Medium ver. 2 (CosmoBio, 
Tokyo, Japan) and cultured in a humidified atmosphere of 5\% CO2. The medium was changed every other day. The cells were cultured for 5 days and then used for subsequent experiments. The lipid droplets were stained with Nile Red (Sigma) and observed under a FV10i confocal microscope (Olympus).

\section{SDS-PAGE and immunoblotting}

SDS-PAGE and immunoblotting were performed according to standard methods described previously [17], anti-UCP1 goat IgG (Santa cruz, CA, USA) and anti-HPRT Rabbit IgG (Santa cruz) were using as the primary anti-body. Anti-goat IgG-HRP and anti-rabbit IgG-HRP (Santa cruz) were using as the secondary antibody. Bands were detected by a Chemi-Lumi one (Nacalai Tesque, Kyoto, Japan).

\section{Statistical analysis}

Data were expressed as means $\pm \mathrm{s}$. d. Student's t-test and two-way ANOVA were used for comparison of significant differences. $P$ values $<0.05$ were considered statically significant. All statically analysis was performed using SAS (version 2.0; Esumi, Tokyo, Japan).

\section{Results}

\section{Sms 2 deficiency impedes body weight gain and adipose tissue hypertrophy.}

To examine the contribution of WAT and BAT in the suppression of diet-induced obesity and insulin resistance in $S m s 2 \mathrm{KO}$ mice, we fed WT and Sms 2 KO mice with a high-fat diet for 12 weeks as previously described $[17,19]$.

The body weights of WT mice fed a high-fat diet increased significantly compared with Sms $2 \mathrm{KO}$ mice. In addition, the body weights of WT mice fed a normal diet were moderately higher than those of $S m s 2$ KO mice (Figure 1A).

The average amounts of food intake were not different between Sms2 KO and WT mice (Figure 1B). These results are consistent with our previous report [17].

The weights of the liver, kidneys, and adipose tissues from WT and Sms2 KO mice of both diet groups were recorded. Although the weights of the liver and kidneys from Sms2 KO mice were comparable with those from WT mice (Table 1), the weights of epididymal and subcutaneous WAT and interscapular BAT were significantly lower in Sms2 KO mice than in WT mice (Figure 1C and Table 1).

In addition, body WAT and BAT percentages decreased in $S m s 2 \mathrm{KO}$ mice compared with WT mice (Figure 1D). These results suggest that SMS2 is involved in adipose tissue hypertrophy, possibly due to the accumulation of neutral lipids. Sms2 deficiency prevents low-grade inflammation and macrophage infiltration in WAT.

We performed detailed histological analysis of adipose tissues from WT and Sms2 KO mice (Figure 2A). The adipocyte area $(2555 \mu \mathrm{m} 2)$ was $\sim 19 \%$ less in Sms2 KO mice fed a normal diet compared with the adipocyte area $(3156.3 \mu \mathrm{m} 2)$ in WT mice (Figure $2 \mathrm{~B}$ ). As shown in Figure 2B, the frequency of small adipocytes increased in Sms $2 \mathrm{KO}$ mice fed a normal diet.

This tendency was also seen in Sms2 KO mice fed a high-fat diet (Figure $2 \mathrm{~B})$. In addition, the adipocyte area $(5260 \mu \mathrm{m} 2)$ was $\sim 30 \%$ less in $\operatorname{Sms} 2 \mathrm{KO}$ mice compared with the adipocyte area $(7560.8 \mu \mathrm{m} 2)$ in WT mice.

Adipocyte hypertrophy associates with inflammation and increased macrophage infiltration [20,21]. In epididymal WAT from WT mice fed a high-fat diet, the expression levels of tumor necrosis

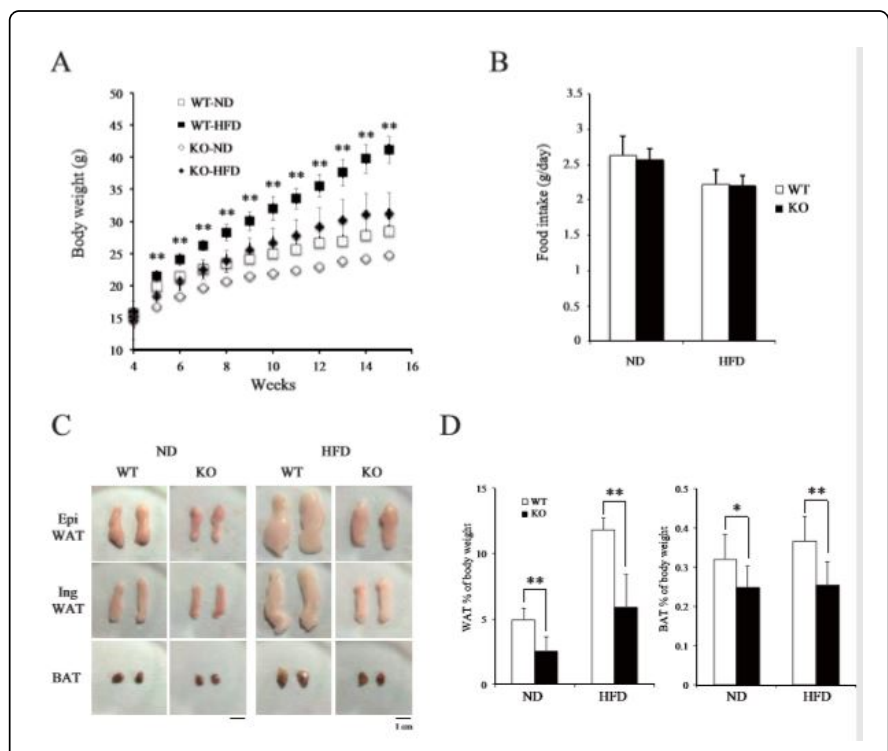

Figure 1: Sms2 deficiency inhibits diet-induced body weight gain and adiposity. (A) Body weights of WT and Sms2 KO mice fed a normal (ND) or high-fat diet (HFD) were obtained weekly $(\mathrm{n}=10$ 16 for each experimental group, ${ }^{*}, 0.01<\mathrm{p}<0.05,{ }^{* *}, \mathrm{p}<0.01$ between WT mice fed the high-fat diet and Sms 2 KO mice fed the high-fat diet). The results are expressed as the means \pm S.D. (B) Dialy food intake of WT and Sms $2 \mathrm{KO}$ mice fed a normal or high-fat diet was recorded in 8-week-old mice ( $\mathrm{n}=9-11$ for each experimental group, $\left.{ }^{*}, 0.01<\mathrm{p}<0.05,{ }^{* *}, \mathrm{p}<0.01\right)$. The results are expressed as the means \pm S.D. (C) Epididymal (Epi), inguinal (Ing), and brown adipose tissues (BAT) in WT and Sms2 KO mice fed a normal or high-fat diet at 15 weeks of age. Scale bar $=1 \mathrm{~cm}$. (D) Percentage of WAT (epididymal and inguinal WAT) and BAT in WT and Sms2 KO mice fed a normal or high-fat diet at 15 weeks of age was calculated $\left(\mathrm{n}=11-13\right.$ for each experimental group, $\left.{ }^{\star}, 0.01<\mathrm{p}<0.05,{ }^{*}, \mathrm{p}<0.01\right)$. The results are expressed as the means \pm S.D.

factor a (Tnfa), monocyte chemotactic protein 1(Mcp1), and the mature macrophage marker $\mathrm{F} 4 / 80$ were elevated, while the adiponectin expression level decreased (Figure 2C and Supplementry Figure 1A).

However, Sms2 deficiency suppressed the increase in the expression levels of Tnfa, Mcp1, and F4/80. In addition, there was a small but statistically significant increase in adiponectin expression (Figure 2C and Supplementry Figure 1A).

There were no changes in the expression levels of these genes between WT and Sms2 KO mice fed a normal diet. To evaluate macrophage infiltration in WAT, we observed macrophages surrounding dead adipocytes, which form characteristic crown-like structures (CLSs).

Although the number of CLSs was not significantly different between WT and Sms2 KO mice fed a normal diet, the number of CLSs was significantly reduced in Sms2 KO mice fed a high-fat diet 
(Figure 2D). These results indicate that obesity-induced inflammation and infiltration of macrophages in WAT may be inhibited by Sms 2 deficiency.

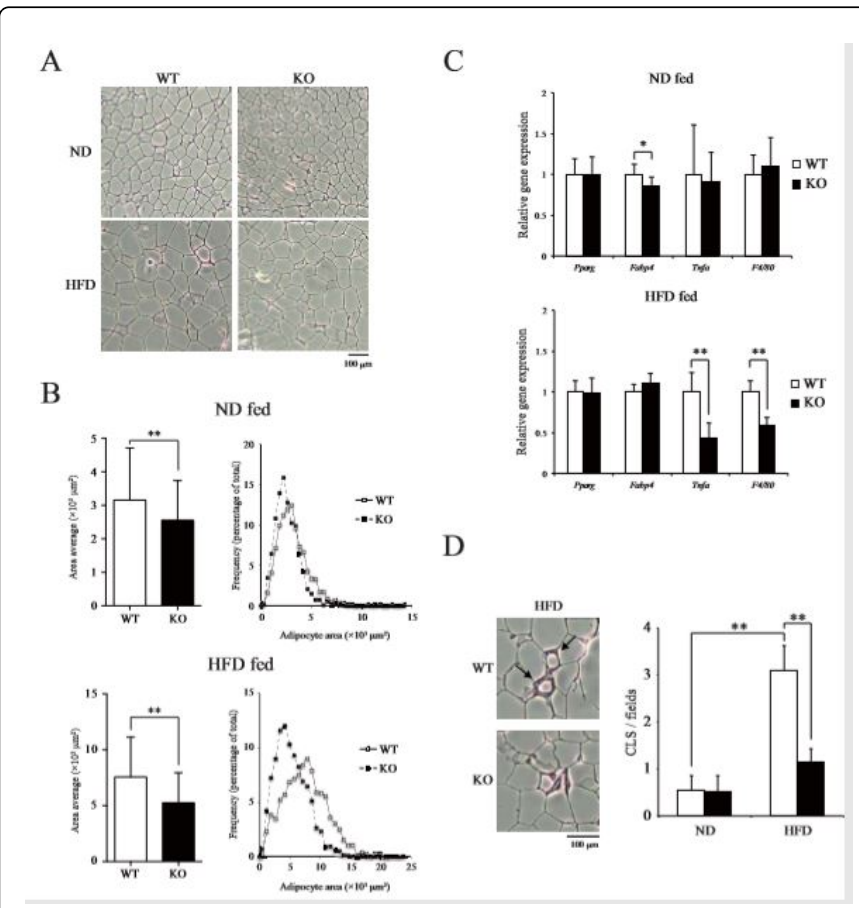

Figure 2: Diet-induced adipocyte hypertrophy and low-grade inflammation are suppressed in epididymal adipose tissue from Sms2 KO mice fed a high-fat diet. (A) Epididymal adipose tissue sections were stained with hematoxylin and eosin. Scale bar=100 am. (B) Surface area and frequency distribution of adipocytes in epididymal WAT from WT and Sms2 KO mice. Adipocyte surface area was measured with ImageJ software, and the distributions were plotted ( $n=4-5$ mice per group, with $>300$ cells counted per mouse). (C) Expression of genes involved in differentiation and inflammation (Fabp4, Pparg Tnfa, F4/80) in epididymal adipose tissue was measured by quantitative real-time PCR $(n=5-8$ mice per group, $\left.{ }^{\star}, 0.01<\mathrm{p}<0.05,{ }^{*}, \mathrm{p}<0.01\right)$. The results are expressed as the means \pm S.D. (D) Crown-like structures (CLSs, arrows) in epididymal adipose tissue were counted ( $n=5-7$ mice per group). Dead adipocytes surrounded by macrophages were quantified in more than five fields per mouse. The results are expressed as the means \pm S.D. Scale bar $=100 \alpha \mathrm{m} .{ }^{\star}, 0.01<\mathrm{p}<0.05,{ }^{*}, \mathrm{p}<0.01$.

\section{Sms2 deficiency moderately inhibits adipocyte differentiation}

As shown in Figure 2C, the expression of fatty acid-binding protein 4 (Fabp4), a marker of adipocyte differentiation, decreased moderately in Sms 2 KO mice fed a normal diet. Compared with WT mice, the expression levels of Fabp4 and peroxisome proliferator-activated receptor $\gamma$ (Pparg), a key transcription factor involved in adipocyte differentiation, also decreased in Sms2 KO mice at 8 weeks of age (Supplementry Figure B), indicating that Sms2 deficiency reduces adipocyte differentiation. To examine the effects of $S m s 2$ deficiency on adipocyte differentiation, we isolated and cultured SVCs from epididymal adipose tissue to induce differentiation into adipocytes.
SVCs isolated from WT mice differentiated into adipocytes with numerous lipid droplets (Figure 3A). Although SVCs isolated from Sms2 KO mice differentiated into adipocytes, they contained many small lipid droplets (Figure 3A). In SVCs isolated from Sms $2 \mathrm{KO}$ mice, the adipocytes containing lipid droplets with a diameter $<2$ am increased, while the adipocytes containing lipid droplets with a diameter $>4$ am decreased (Figure 3B). We also examined the expression levels of genes involved in adipocyte differentiation. In differentiated SVCs isolated from Sms2 KO mice, Pparg expression decreased significantly, and the expression levels of its downstream targets that included Fabp4, glucose transporter type 4 (Glut4), fatspecific protein $27 \mathrm{kDa}$ (Fsp27), perilipin 1 (Plin1), adipose triglyceride lipase (Atgl), and hormone sensitive lipase (Hsl) also decreased (Figure 3C). These results suggest that the adipocyte differentiation is moderately inhibited by $S m s 2$ deficiency.
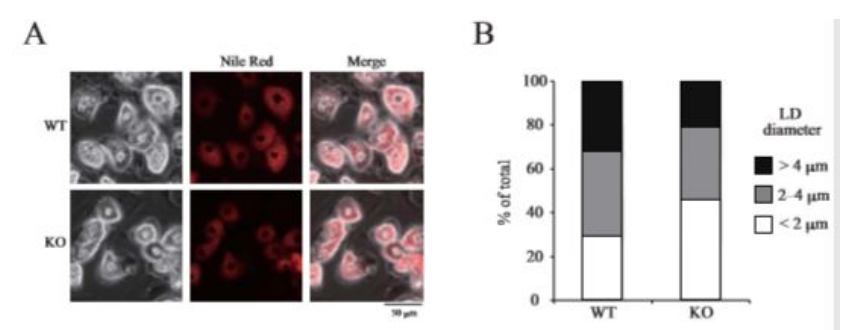

C

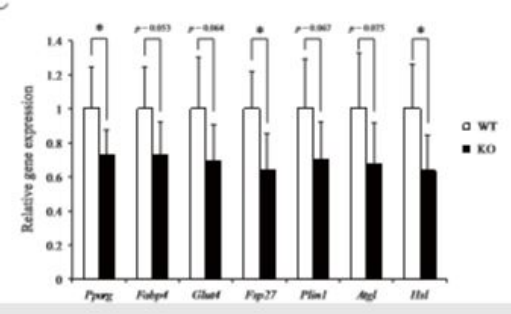

Figure 3: Sms2 deficiency moderately suppresses differentiation. (A) Preadipocytes isolated from epididymal adipose tissue were cultured to induce differentiation and then stained with Nile Red. Scale bar=50 am. (B) Frequency distribution of the diameters of lipid droplets (LD) for each class of adipocytes (LD diameter, $>4$ am, 2-4 am, and $<2$ am). More than 100 Nile Red-positive cells were counted in each experiment. (C) Expression of genes involved in adipocyte differentiation (Fabp4, Pparg Glut4), lipid droplet formation/fusion (Fsp27 and Plin1), and lipid metabolism (Atgl and Hsl) was measured by quantitative real-time PCR. The results are expressed as the means \pm S.D. ${ }^{\star}, 0.01<\mathrm{p}<0.05$, ${ }^{\star *}, \mathrm{p}<0.01$. All experiments were independently repeated at least three times.

\section{Sms2 deficiency is involved in the browning of subcutaneous WAT}

To examine the effects of Sms2 deficiency on subcutaneous adipose tissue, we performed histological analysis of adipose tissue from WT and Sms2 KO mice (Figure 4A).

Histological analysis revealed that the surface area of adipocytes from $\operatorname{Sms} 2 \mathrm{KO}$ mice fed a normal or high-fat diet decreased compared to WT mice (Figure 4A, B). In addition, the number of small adipocytes increased in Sms2 KO mice fed a normal or high-fat diet compared to WT mice (Figure 4A, 4B). 
The results from this analysis were comparable to those from the epididymal adipose tissue analysis (Figures $2 \mathrm{~A}$ and $2 \mathrm{~B}$ ). However, the expression levels of Fabp4 and Pparg in subcutaneous WAT were not different between WT and Sms2 KO mice (Figure 4C).

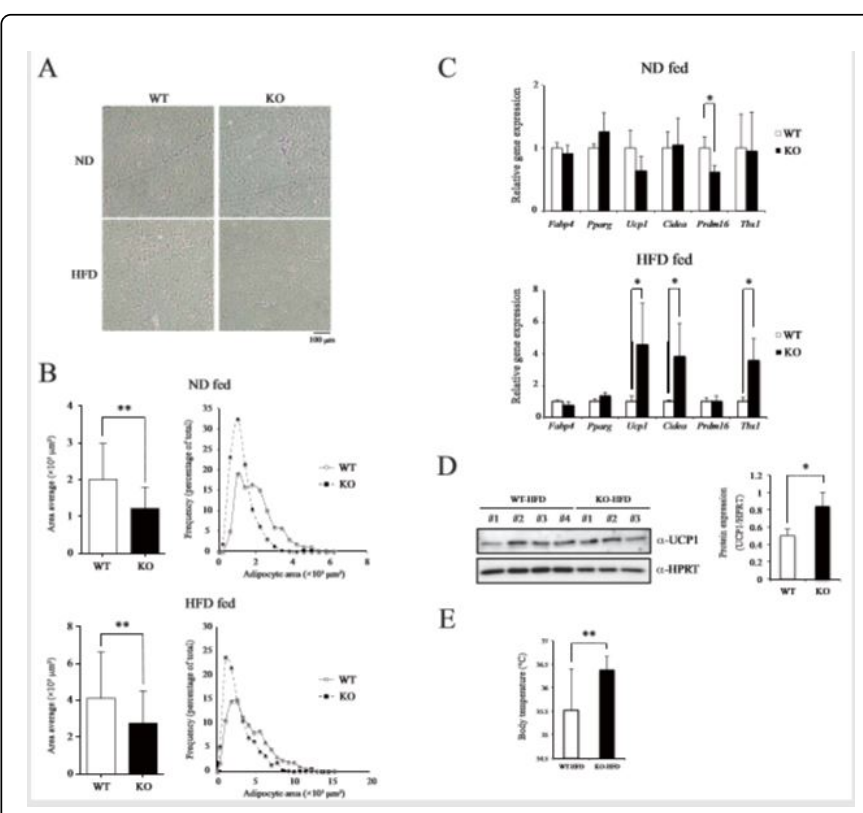

Figure 4: Expression of genes involved in energy expenditure and browning in subcutaneous adipose tissue from Sms2 KO mice fed a high-fat diet is increased. (A) Inguinal adipose tissue sections were stained with hematoxylin and eosin. Scale bar=100 am. (B) Surface area and frequency distribution of adipocytes in inguinal WAT from WT and $S m s 2 \mathrm{KO}$ mice. Adipocyte surface area was measured with ImageJ software, and the distributions were plotted ( $\mathrm{n}=3$ mice per group, with $>180$ cells counted per mouse). (C) Expression of genes involved in differentiation (Fabp4 and Pparg, as well as the expression of brown adipocyte markers (Ucp1, Cidea, Prdm16) and the beige adipocyte marker Tbx1 in inguinal adipose tissue, was measured by quantitative real-time PCR ( $\mathrm{n}=3-6$ mice per group, ${ }^{*}$, $0.01<\mathrm{p}<0.05,{ }^{* *}, \mathrm{p}<0.01$ ). (D) Immunoblotting of UCP1. UCP1 protein expression was normalized loading control HPRT1. (E) Body temperature of WT and Sms $2 \mathrm{KO}$ mice fed a high fat-diet at 16 weeks of age $\left(n>9,{ }^{* *}, p<0.01\right)$. The results are expressed as the means \pm S.D.

Subcutaneous WAT browning associates with increased energy expenditure, which decreases obesity and improves glucose intolerance $[5,6]$.

Thus, we investigated the expression level of the beige adipocyte marker Tbx1, as well as the expression levels of genes involved in thermogenesis (uncoupling protein 1 (Ucp1), cell death-inducing DFFA-like effector A (Cidea), PR domain 16 (Prdm16)). Except for Prdm16, there were no changes in their expression levels between WT and $S m s 2 \mathrm{KO}$ mice fed a normal diet.

On the other hand, the expression levels of Ucp1, Cidea, and Tbx1 increased significantly in Sms $2 \mathrm{KO}$ mice fed a high-fat diet (Figure $4 \mathrm{C})$.

In addition, Ucp1 protein expression level was slightly but significantly increased (Figure 4D). In concordance with the elevated expression of Ucp1, the rectal temperature was higher in Sms $2 \mathrm{KO}$ mice fed a high-fat diet than in WT mice fed a high-fat diet (Figure $4 \mathrm{E})$. These results indicate that the browning of subcutaneous WAT is induced in Sms2 KO mice.

\section{Fatty acid synthesis is suppressed in BAT of $S m s 2$ KO mice}

We performed histological analysis of BAT from WT and Sms 2 KO mice. There were many multilocular lipid droplets present in BAT from WT mice fed a normal diet. However, the lipid droplets underwent hypertrophy in WT mice fed a high-fat diet (Figure 5A). In BAT from Sms $2 \mathrm{KO}$ mice, the lipid droplets were very small, and an increase in surface area was inhibited by a high-fat diet (Figure 5A). Thus, we hypothesized that energy expenditure was induced or/and brown adipocyte differentiation was suppressed in $S m s 2 \mathrm{KO}$ mice. To test this hypothesis, we investigated the expression levels of genes involved in

adipocyte differentiation (Fabp4 and Pparg, glucose uptake (Glut4), and energy expenditure (Ucp1, Cidea, Prdm16). However, there were no changes in their expression levels between WT and Sms2 KO mice (Figure 5B). We also investigated the expression levels of genes involved in. fatty acid $\quad \beta$-oxidation and synthesis (acyl-CoA oxidase 1 (Acox 1$)$, carnitine palmitoyltransferase $1 \mathrm{~b}(\mathrm{Cpt} 1 \mathrm{~b})$, fatty acid synthase (Fasn), stearoyl-coenzyme A desaturase 1 (Scd1)). Compared to WT mice fed normal and high-fat diets, the expression levels of Acox 1 and Cpt1b were similar in BAT from Sms2 KO mice fed normal and highfat diets.

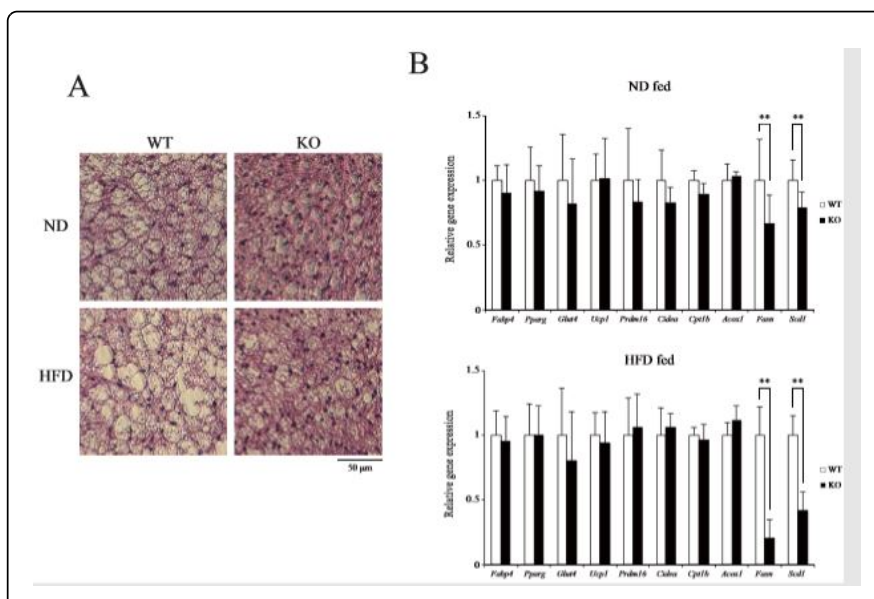

Figure 5: Fatty acid synthesis is suppressed in BAT from Sms2 KO mice fed normal and high-fat diet. (A) Brown adipose tissue sections were stained with hematoxylin and eosin. Scale bar=50 am. (B) Expression of genes involved in differentiation (Fabp4 and Pparg, and fatty acid metabolism and synthesis (Cpt1, Acox1, Fasn, Scd1), as well as the expression of brown adipocyte markers (Ucp1, Cidea, Prdm16), was measured by quantitative real-time PCR $\left(\mathrm{n}=5-13\right.$ mice per group, $\left.{ }^{*}, 0.01<\mathrm{p}<0.05,{ }^{* *}, \mathrm{p}<0.01\right)$. The results are expressed as the means \pm S.D.

However, the expression levels of Fasn and Scd1 decreased significantly in BAT from Sms $2 \mathrm{KO}$ mice fed normal and high-fat diets (Figure 5B). These results indicate that fatty acid synthesis is suppressed in BAT from Sms2 KO mice but that fatty acid oxidation is normal. 


\section{Discussion}

Mitsutake et al. reported that Sms2 KO mice fail to develop dietinduced obesity, systemic insulin resistance, and liver steatosis [17]. However, there is no study on the roles of WAT and BAT, which associate with the development of metabolic diseases, in Sms2 KO mice. In this study, we investigated whether WAT and BAT from Sms2 $\mathrm{KO}$ mice contribute to the suppression of diet-induced obesity and insulin resistance. The body weights of Sms $2 \mathrm{KO}$ mice, as well as the weights of different adipose tissues, were reduced compared with WT mice. High-fat diet-induced adipocyte hypertrophy and low-grade inflammation were also inhibited in epididymal WAT from Sms $2 \mathrm{KO}$ mice. Moreover, adipocyte differentiation was moderately inhibited in Sms2-deficient white adipocytes. Energy expenditure increased by the recruitment and activation (browning) of beige adipocytes in subcutaneous WAT from Sms 2 KO mice. In BAT from Sms2 KO mice, fatty acid synthesis was inhibited. These findings indicate that Sms2 deficiency in adipose tissues suppresses diet-induced obesity and insulin resistance.

Several studies reported that adipocyte hypertrophy increases the surface area of visceral adipose tissue in diet-induced or genetically obese mice, leading to the development of low-grade inflammation $[24,25]$. However, the increased adipose tissue weight, the adipocyte hypertrophy, the macrophage infiltration, and the expression levels of inflammatory cytokines and chemokines (Figure 2) were inhibited in epididymal adipose tissue from Sms2 KO mice. Hailemariam et al. reported that the activation of nuclear factor-kappa $\mathrm{BNF \kappa B}$ ), a central protein in the inflammatory response, was attenuated in macrophages isolated from Sms2 KO mice [26]. An inhibition of chronic inflammation in adipose tissue from obese subjects can improve insulin sensitivity via an increase in serine/threonine-protein kinase (Akt) phosphorylation $[27,28]$. Indeed, Sugimoto et al. reported that Akt phosphorylation increased in visceral adipose tissue from Sms2 KO mice [29]. Therefore, the improvement of insulin sensitivity in adipose tissue from $S m s 2 \mathrm{KO}$ mice via an inhibition of inflammation may contribute to the suppression of systemic insulin resistance. The surface area of white adipocytes from $\operatorname{Sms} 2 \mathrm{KO}$ mice was lower than that of WT mice fed normal and high-fat diets (Figure 2). Moreover, the expression levels of Pparg and Fabp4 decreased in Sms2 KO mice (Figure 2 and Supplementry Figure 1B). Consistent with these results, preadipocytes isolated from WAT from $\mathrm{Sms} 2 \mathrm{KO}$ mice matured slower than those isolated from WAT from WT mice (Figure 3). The body weights, as well as the weights of adipose tissues, decrease in adipose tissue-specific Pparg KO mice, because adipocyte differentiation is insufficient [30]. These results reveal that moderate defects in adipocyte differentiation in Sms2 KO mice underlie the reduction in adipose tissue mass and the increased number of small adipocytes. Although the food intake in WT and Sms 2 KO mice fed a high-fat diet was not changed, the body weights of $\operatorname{Sms} 2 \mathrm{KO}$ mice fed a high-fat diet were significantly lower than those of WT mice (Figure 1). These results led us to conclude that energy expenditure is increased in Sms2 $\mathrm{KO}$ mice. A recent study reported an increase in energy expenditure during the recruitment of beige adipocytes in subcutaneous adipose tissue but a decrease in diet-induced obesity [6]. Furthermore, it is reported that many of genetic modification mice that resist to dietinduced obesity showed that browning in subcutaneous adipose tissue [6, 31-35]. In subcutaneous adipose tissue from Sms2 KO mice fed a high-fat diet, the expression levels of genes involved in browning (Ucpl and Cidea), as well as the expression level of the beige adipocyte marker gene Tbx1, increased compared to WT mice (Figure 4). These results raise the possibility that the browning of subcutaneous fat in
Sms2 KO mice may be one of the causes of diet-induced obesity resistance. However, other explanations also exist such as poor intestinal absorption of triglycerides or hyperactivity. The intestinal absorption of triglycerides (TGs) in Sms2 KO mice may have few effects, because the fecal TGs contents in Sms2 KO mice were comparable to those in WT mice (data not shown). To evaluate hyperactivity in Sms2 KO mice, however, the voluntary activity will have to be measured. It is also important to investigate the effects of Sms 2 deficiency on sympathetic activity, because sympathetic neurons and neuropeptides are involved in the regulation of adipose tissue function [36-38].

Lodhi et al. reported that Fasn deficiency in adipose tissue increased energy expenditure, resulting in subcutaneous adipose tissue browning and a lean phenotype [23]. The authors also indicated that alkyl ether phosphatidylcholine species, which act as PPAR $\gamma$ agonists, decreased in adipose tissue-specific Fasn KO mice. In Sms2 KO mice, on the other hand, Fasn expression in BAT decreased significantly (Figure 5). In addition, the expression levels of genes involved in browning (Ucp1, Cidea, Tbx1) increased in subcutaneous adipose tissue (Figure 4). Recently, Xia et al. reported that dynamic crosstalk exists between sphingolipids in the liver and adipose tissue, and that this bidirectional transport of sphingolipids is important for the regulation of glucose metabolism and hepatic lipid uptake. Moreover, an alteration of sphingolipid composition in adipose tissues induced a quicker and stronger resolution of hepatic steatosis than that of sphingolipid composition in the liver [39]. Although we have not yet analyzed the phospholipid and sphingolipid composition in adipose tissue, changes in the composition of phospholipids and sphingolipids in adipose tissues from Sms2 KO mice might contribute to the browning of subcutaneous fat, and the suppression of liver steatosis and insulin resistance in these mice. To address these possibilities, the composition of different lipids should be analyzed in tissue-specific conditional Sms2 KO mice. SMS1, an isoform of SMS, localizes to the Golgi apparatus. It is responsible for synthesizing most of the SM [13]. Similar to $S m s 2$ KO mice, Sms1 KO mice exhibited a lean phenotype and a decreased WAT weight. However, mitochondrial dysfunction and increased oxidative stress can affect adipose tissue function, subsequently resulting in hypertriglyceridemia (lipodystrophy) $[15,16]$. On the other hand, Sms2 KO mice exhibited a decreased WAT weight (Figure 1), but not hypertriglyceridemia [17]. There were no changes in the expression levels of genes involved in mitochondrial function (Acox1, Cpt1b, Ucp1) in WT mice fed a normal diet (Figure 5). Moreover, oxidative stress derived from mitochondrial disfunction in adipose tissues was not changed between WT and Sms2 KO mice (Supplementry Figure 2). Therefore, the mitochondrial function in adipose tissues from Sms2 KO may be normal. In contrast to SMS1, SMS2 localizes predominantly to the plasma membrane, and it is important for the maintenance of SM levels in lipid rafts [12-14,17]. Thus, the regulation of SM levels or the maintenance of lipid rafts in the plasma membrane via SMS2 activity may be involved in adipocyte differentiation, inflammation, and browning, as well as the regulation of the fatty acid synthesis pathway.

\section{Conclusion}

Sms2 deficiency leads to moderate defects in adipocyte differentiation and the suppression of inflammation in visceral fat, increased energy expenditure by the browning of subcutaneous fat, and the suppression of fatty acid synthesis in BAT. These synergetic effects in adipose tissues caused by $S m s 2$ deficiency may contribute to 
the suppression of diet-induced obesity and insulin resistance. Additional studies are needed to precisely define the role of SMS2 in adipose tissue.

\section{Acknowledgment}

This work was supported by the Creation of Innovation Centers for Advanced Interdisciplinary Research Areas Program of the Ministry of Education, Culture, Sports, Science and Technology, Japan. We thank Dr. Takamitsu Sano for technical advice. We also thank Dr. Jun-ichi Furukawa, Dr. Tetsuo Mioka, and the members of the Igarashi laboratory for valuable discussions.

\section{Conflict of Interest}

The authors declare no conflict of interest.

\section{References}

1. Peirce V, Carobbio S, Vidal-Puig A (2014) The Different Shades of Fat. Nature 510: 76-83.

2. Lelliott C, Vidal-Puig AJ (2004) Lipotoxicity, an Imbalance between Lipogenesis De Novo and Fatty Acid Oxidation. Int J Obes Relat Metab Disord. 28: S22-S28.

3. Cannon B, Nedergaard J (2004) Brown Adipose Tissue: Function and Physiological Significance. Physiol Rev 84: 277-359.

4. Lowell BB, Spiegelman BM (2000) Towards A Molecular Understanding of Adaptive Thermogenesis. Nature 404: 652-660.

5. Wu J, Bostrom P, Sparks LM, Ye L, Choi JH, et al. (2012) Beige Adipocytes are a Distinct Type of Thermogenic Fat Cell in Mouse and Human. Cell 150: 366-376.

6. Seale P, Conroe HM, Estall J, Kajimura S, Frontini A (2011) Prdm16 Determines the Thermogenic Program of Subcutaneous White Adipose Tissue in Mice. J Clin Invest 121: 96-105.

7. Hannun YA, Obeid LM (2008) Principles of Bioactive Lipid Signalling Lessons from Sphingolipids. Nat Rev Mol Cell Biol 9: 139-150.

8. Zheng W, Kollmeyer J, Symolon H, Momin A, Munter E, et al. (2006) Ceramides and Other Bioactive Sphingolipid Backbones in Health and Disease: Lipidomic Analysis, Metabolism and Roles in Membrane Structure, Dynamics, Signaling and Autophagy. Biochim Biophys Acta 1758: 1864-84.

9. Chaurasia B, Summers SA (2015) Ceramides-Lipotoxic Inducers of Metabolic Disorders. Trends Endocrinol Metab 26: 538-550.

10. Yuyama K, Mitsutake S, Igarashi Y (2014) Pathological Roles of Ceramide and Its Metabolites in Metabolic Syndrome and Alzheimer's Disease. Biochim Biophys Acta 1841: 793-788.

11. He X, Huang Y, Li B, Gong CX, Schuchman EH (2010) Deregulation of Sphingolipid Metabolism in Alzheimer's Disease. Neurobiol Aging 31: 398-408.

12. Ding T, Li Z, Hailemariam T, Mukherjee S, Maxfield FR, et al. (2008) SMS Overexpression and Knockdown: Impact on Cellular Sphingomyelin and Diacylglycerol Metabolism, And Cell Apoptosis. J Lipid Res 49: 376-385.

13. Tafesse FG, Ternes P, Holthuis JC (2006) The Multigenic Sphingomyelin Synthase Family. J Biol Chem 281: 29421-29425.

14. Li Z, Hailemariam TK, Zhou H, Li Y, Duckworth DC, et al. (2007) Inhibition of Sphingomyelin Synthase (SMS) Affects Intracellular Sphingomyelin Accumulation and Plasma Membrane Lipid Organization. Biochim Biophys Acta 17719:1186-1194.

15. Yano M, Watanabe K, Yamamoto T, Ikeda K, Senokuchi T, et al. (2011) Mitochondrial Dysfunction and Increased Reactive Oxygen Species Impair Insulin Secretion in Sphingomyelin Synthase 1-Null Mice. J Biol Chem 286: 3992-4002.
16. Yano M, Yamamoto T, Nishimura N, Gotoh T, Watanabe K, et al. (2013) Increased Oxidative Stress Impairs Adipose Tissue Function in Sphingomyelin Synthase 1 Null Mice. PLoS One 8: e61380.

17. Mitsutake S, Zama K, Yokota H, Yoshida T, Tanaka M, Mitsui M et al. (2011) Dynamic Modification of Sphingomyelin in Lipid Microdomains controls development of Obesity, Fatty liver, and type 2 diabetes. J Biol Chem 286: 28544-28545.

18. Sato T, Nagafuku M, Shimizu K, Taira (2008) Physiological levels of insulin and IGF-1 differentiation of mesenteric adipocytes. Cell Bio Int 32: 1397-1404

19. Weisberg SP, McCann D, Desai M, Rosenbaum M, Leibel RL, et al. (2003) Obesity is associated with Macrophage Accumulation in Adipose Tissue. J Clin Invest 112: 1796-1808.

20. Harman-Boehm I, Bluher M, Redel H, Sion-Vardy N, Ovadia S, et al. (2007) Macrophage Infiltration into Omental Versus Subcutaneous Fat across different Populations: Effect of Regional Adiposity and the Comorbidities of Obesity. J Clin Endocrinol Metab 92: 2240-2247.

21. Lee J, Ellis JM, Wolfgang MJ (2015) Adipose Fatty Acid Oxidation is required for Thermogenesis and Potentiates oxidative stress-induced inflammation. Cell Rep 10: 266-279.

22. Lodhi IJ, Yin L, Jensen-Urstad AP, Funai K, Coleman T, et al.(2012) Inhibiting Adipose Tissue Lipogenesis Reprograms Thermogenesis and Ppary activation to Decrease Diet-Induced Obesity. Cell Metab 16: 189-20.

23. Faust IM, Johnson PR, Stern JS, Hirsch J (1987) Diet-Induced Adipocyte Number Increase in Adult Rats: A New Model of Obesity. Am J Physiol 235: E279-E286.

24. Hotamisligil GS, Shargill NS, Spiegelman BM (1993) Adipose Expression of Tumor Necrosis Factor-Alpha: Direct Role in Obesity-Linked Insulin Resistance. Science 259: 87-91.

25. Hailemariam TK, Huan C, Liu J, Li Z, Roman C, et al. (2008) Sphingomyelin Synthase 2 Deficiency Attenuates Nfkappab Activation. Arterioscler Thromb Vasc Biol 28: 1519-26.

26. Uysal KT, Wiesbrock SM, Marino MW, Hotamisligil GS (1997) Protection from Obesity-Induced Insulin Resistance in Mice Lacking TNF-Alpha Function. Nature 389: 610-614

27. Shao J, Yamashita H, Qiao L, Friedman JE (2000) Decreased Akt Kinase Activity and Insulin Resistance in C57bl/Ksj-Leprdb/Db Mice. J Endocrinol 167: 107-115.

28. Sugimoto M, Shimizu Y, Zhao S, Ukon N, Nishijima KI, et al. (2016) Characterization of the Role of Sphingomyelin Synthase 2 in Glucose Metabolism in Whole-Body and Peripheral Tissues in Mice. Biochim Biophys Acta 1861: 688-702.

29. Jones JR, Barrick C, Kim KA, Lindner J, Blondeau B, et al. (2005) Deletion of Ppargamma in Adipose Tissues of Mice Protects Against High Fat Diet-Induced Obesity and Insulin Resistance. Proc Natl Acad Sci 102: 6207-6212.

30. Sawada T, Miyoshi H, Shimada K, Suzuki A, Okamatsu-Ogura Y, et al. (2010) Perilipin Overexpression in White Adipose Tissue Induces a Brown Fat-Like Phenotype. PLoS One 5: e14006.

31. Cui XB, Luan JN, Ye J, Chen SY (2015) Rgc32 Deficiency protects against High-Fat Diet-Induced Obesity and Insulin Resistance in Mice. J Endocrinol 224: 127-137.

32. Chiang SH, Bazuine M, Lumeng CN, Geletka LM, Mowers J, et al. (2009) The Protein Kinase IKKepsilon regulates Energy Balance in Obese Mice. Cell 138: 961-975.

33. Toh SY, Gong J, Du G, Li JZ, Yang S, et al. (2008) Up-Regulation of Mitochondrial Activity and Acquirement of Brown Adipose Tissue-Like Property in the White Adipose Tissue of Fsp27 Deficient Mice. PLoS One 3: e2890.

34. Leonardsson G, Steel JH, Christian M, Pocock V, Milligan S, et al. (2004) Nuclear Receptor Corepressor Rip140 Regulates Fat Accumulation. Proc Natl Acad Sci 101: 8437-8442. 
Citation: Hanamatsu H, Mitsutake S, Sakai S, Okazaki T, Watanabe K, et al. (2018) Multiple Roles of Sms2 in White and Brown Adipose Tissues from Diet-induced Obese Mice. J Metabolic Synd 7: 241. doi:10.4172/2167-0943.1000241

Page 8 of 8

35. Bamshad M, Aoki VT, Adkison MG, Warren WS, Bartness TJ (1998) Central Nervous System Origins of the Sympathetic Nervous System Outflow to White Adipose Tissue. Am J Physiol 275: R291-R299.

36. Youngstrom TG, Bartness TJ (1995) Catecholaminergic Innervation of White Adipose Tissue in Siberian Hamsters. Am J Physiol 268: R744R751.

37. Ohta H, Konishi M, Kobayashi Y, Kashio A, Mochiyama T, et al. (2015) Deletion of the Neurotrophic Factor Neudesin Prevents Diet-Induced Obesity by Increased Sympathetic Activity. Sci Rep 5: 10049.
38. Xia JY, Holland WL, Kusminski CM, Sun K, Sharma AX, et al. (2015) Targeted Induction of Ceramide Degradation Leads to Improved Systemic Metabolism and Reduced Hepatic Steatosis. Cell Metab 22: 266-78. 